\title{
The Gastric Bezoar Revealed by an Acute Intestinal Obstruction: An Observation and Literature Review
}

\author{
R Berraida ${ }^{1 *}$, S Mrabti ${ }^{1}$, S Berrag ${ }^{1}$, I Elkoti ${ }^{1}$, M Tamzaourt ${ }^{1}$, H Seddik ${ }^{1}$, A Benkirane ${ }^{1}$ and \\ F Alazaoui A Eratbi 0 Lahnaoui \\ ${ }^{1}$ Gastroenterology Department II, Military Hospital Mohamed V, Rabat, Morocco \\ ${ }^{2}$ Surgical Deparment, avicenne Hospital in Rabat \\ *Corresponding Author: R Berraida, Gastroenterology Department II, Military Hospital Mohamed V, Rabat, Morocco.
}

Received: July 23, 2019; Published: August 14, 2019

\begin{abstract}
Introduction: "Bezoar" refers to a rare condition, secondary to unusual accumulation, in the form of solid masses or concretions, of substances of various kinds inside the digestive tract and more particularly, in the stomach. It mainly affects young patients who are disturbed by psychological disorders. We report a case of gastric bezoar through which diagnostic and therapeutic difficulties will be discussed.

Observation: This is a phytobezoar in a 17-year-old woman, revealed by vomiting and epigastralgia complicated by intestinal obstruction, Upper fibroscopy (FOGD) made the diagnosis. The therapeutic management was surgical.
\end{abstract}

Keywords: Bezoar; Stomach; FOGD; Surgery

\section{Introduction}

Trichobezoar is a raredisease that usually occurs in adolescents with mental disorders. Its clinical symptomatology is very varied and the diagnosis is often suspected at radiology and endoscopy. The treatment is essentially surgical and associated with psychological care. We report a case collected in the gastroenterology department of the Mohammed V Military Hospitalin Rabat.

\section{Observation}

This is a 17-year-old girl, with no notable pathological history or notion of trichophagy; for five months she had been experiencing epigastric pain of the type of cramps and post prandial heaviness associated with food vomiting, nauseous eructation with foul breath without intestinal transit disorders, all in a context of unquantified weight loss. The clinical examination was normal with a BMI: 22KG/M2 FOGD (Figure1) showed an intraluminal gastric formation made of intertwined hair mixed with whole-stomach foods corresponding to a trichobezoar with an erythematous antrofundic mucosa and angular ulcerations, the biological assessment showed microcytic hypochrome anemia at $10.4 \mathrm{~g} / \mathrm{dl}$ and a Rate of protein of $62 \mathrm{~g} / \mathrm{l}$. The patient was programmed for endoscopic extraction of the bezoar but became complicated by an occlusive syndrome, ASP had shown colic-like hydroaeric levels, Figure 2.
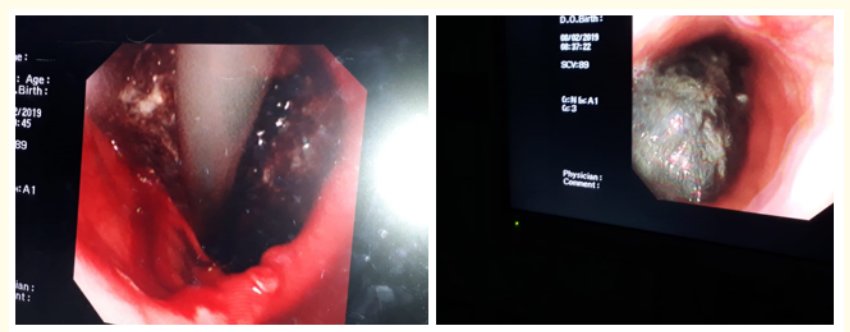

Figure 1: Endoscopic appearance of a gastric trichobezard. 


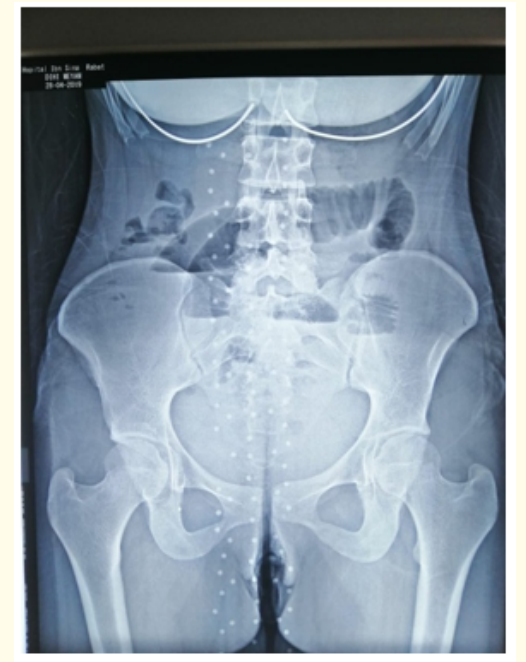

Figure 2: Hydroaeric level of the grélique type.

Therapeutic management consisted of surgical extraction of the trichobezoar by a median incision under umbilical, exploration had shown a distended hail and a foreign body $1 \mathrm{~m}$ from the first intraluminal loop responsible for upstream dilation and peritoneal effusion, moreover it had no anomalies on the remaining hail or on the stomach; (Figure. 3) the operative consequences were simple.
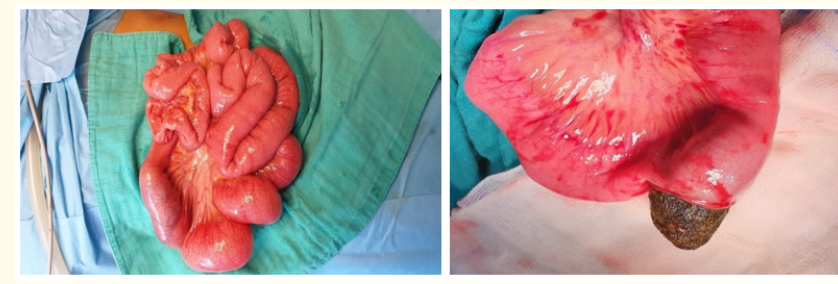

Figure 3: Trichobezoar with an extension in the small intestine characteristic of Rapunzel syndrome.
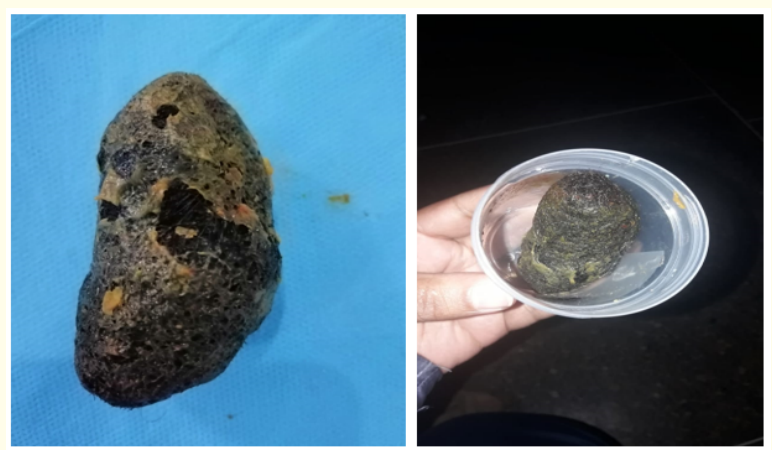

Figure 4: Gastric trichobezoard after surgical extraction.

\section{Discussion}

The term "Bézoard" comes from the Persian panzehr, or the Arabic badzehr, which means antidote or antipoison [1,2]. It refers to a rare condition, secondary to unusual accumulation, in the form of solid masses or concretions, of substances of various kinds inside the digestive tract and more particularly in the stomach, but also sometimes in the urinary tract. The nature of these substances determines the type of bezoar. Thus, the trichobezoar, which represents $55 \%$ of all bezoars, is made of hair, hair or carpet fibers of varying sizes, intertwined with each other, most often in the gastric lumen that can mold it [1].

The female sex is involved in more than $90 \%$ of cases and the age of onset is $80 \%$ of cases under 30 years of age, with a peak incidence between 10 and 19 years old [1]. Trichobezoar is most often observed in emotionally disturbed or depressed patients, psychiatric patients, mentally retarded people and prisoners, who swallow their hair (trichophagy) after pulling it out (trichotillomania) [3]. It can occur spontaneously in hairdressers, wool workers and carpet weavers [1].

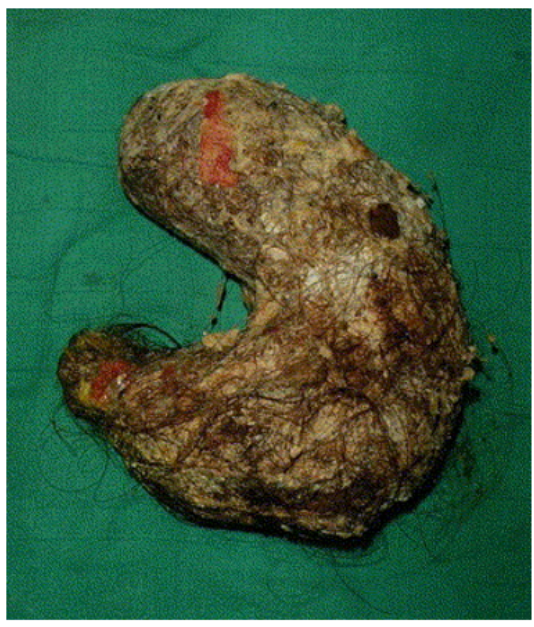

Figure 5: Trichobezoard extracted: its shape is that of the stomach from which it molded the light from which it molded the light.

Classical favoring factors include gastric emptying disorders [4.5], loss of normal motor functions of the pylorus, following partial gastrectomy, and a diet high in fiber [6]. Gastric antisecretory treatments $[7,8]$ appear influencing: the hypoacidity they induce reduces the activity of enzymes (pepsin, cellulase) involved in the disintegration of dietary fiber. Insufficient chewing, due to the absence of teeth, for example, as well as the tachyphagia can lead to 
form ationof bezoars. Eating hair or non-digestible materials, or excessive consumption of food that is difficult to digest can also lead to abezoar. These are rare phenomena, more frequent in the small intestine than in the stomach. the stomach $[7,8,9]$. Psychological disturbances are generally reported: depression, mental retardation, behavioral disorders [10-11] but this is not the case for our patient.

The clinic is not unambiguous. Trichobezoar may remain asymptomatic for a long time or manifest as a vague epigastric gene (80\%), abdominal pain (70\%), nausea or vomiting (65\%), asthenia with weight loss (38\%) ordisorderstransit (33\%) with type of diarrhea or constipation [1,3-5]. A complication may be the mode of revelation of this pathology [5]. It may be upper digestive bleeding due to parietal ulcerations, mechanical gastric or hail occlusion $[6,7]$. Hail occlusion by trichobezoardis very rare, encountered in only $10 \%$ of cases [25] is the case of our patient.

Other complications have been described as gastric or hail perforation with peritonitis or subphrenic abscess [2,7-9], digestive fistula $[9,10]$, cholestasis or acute pancreatitis due to obstruction of the Vater ampoule by an extension of the trichobezoard (Rapunzel syndrome) [11-13]. Some of our patient's history may therefore be related to her bezoar. Other less frequent complications have been reported, such as chronic gastritis, Menetrier disease, gastric polyposis [14], steatorrhea, exudative enteropathy [14] or cystic pneumatosis [15].On clinical examination, in $85 \%$ of cases, there is a well limited, smooth, firm, mobile abdominal mass with epigastric localization; alopecia or foul breath can also be noted [1,12].

Endoscopy is the technique of choice for diagnosing and classifying bezoars. Trichobezoards are black as tar, while phytobezoards are multicoloredranging from yellow to brown or green $[2,10,20,20,21]$. An X-ray of the abdomen may show a mass invading the gastric air bag, but it rarely allows to find the mass involved in the occlusion: risk of confusion with stool or abscess [2]. The scanner and MRI reveal a mass with air pockets without contrast capture $[10,20]$.

Therapeutic management depends on the size and the presence or absence of complications. For a small gastric trichobezoar, it is usually endoscopically extirpatable, the bezoar can be mechanically fragmented using biopsy forceps or polypectomy handles and then removed by washing and suction $[2,20]$. However, this pro- cedure may result in gastric or esophageal perforation reported in some series [20]. Dissolution tests have been performed using papain, acetylcysteine and cellulase, but these rarely succeed [2].

But if it is bulky and heard at the handles or at the complication stage, surgery is the treatment of choice, as was the case with our patient. Laparotomy with extraction of the trichobezoar by gastrotomy, possibly supplemented by enterostomy, is the treatment of extended forms and time-bound cases of endoscopic treatment [3]. Recently, the laparoscopic approach has been proposed as an alternative to laparotomy In addition, psychiatric care, based on behavioural therapy, parental education and medical treatment, must often be provided for patients with trichophagia [5,8]. Our patient had no psychicdisorder.

\section{Conclusion}

The bezoar remains a pathological curiosity, due to its nature and rarity. His diagnosticis certainly easily applied by upper digestive fibroscopy and imaging to highlight other intestinal locations, thus facilitating their therapeutic management. The treatment of choice is the surgery that must be performed urgently to avoid complications of the occlusion. In addition to surgery, psychological care is an essential time in the treatment and especially the prevention of recurrences. Must be performed urgently to avoid complications of the occlusion. In addition to surgery, psychological care is an essential time in the treatment and especially the prevention of recurrences.

\section{Bibliography}

1. DeBakey M and Ochsner A. "Bezoars and concretions: comprehensive review of the literature with analysis of 303 cases and presentation of eight additional cases". Surgery 4 (1938): 934-963.

2. Faria AP., et al. "The Rapunzel syndrome a case report: trichobezoar as a cause of intestinal perforation". Journal of Pediatrics 76.1 (2000): 83-86.

3. Romand F., et al. "Tuberculoseabdominaled'allure pseudo tumorale”. La Presse médicale 26.36 (1997): 1717-1721.

4. Sharma S., et al. "gastric and intestinal trichobezoar a case report". Journal of the Indian Medical Association 102.9 (2004): 516-518. 
5. Stanten A and Peter HE. "Enzymatic dissolution of phytobezoars". The American Journal of Surgery 130.2 (1975): 259261.

6. $\quad$ Rippollés T., et al. "Gastrointestinal Bezoars: sonographic and CT characteristics". American Journal of Roentgenology 177.1 (2001): 65-69.

7. Andrus CH and Ponsky JL. "Bezoars classification, pathophysiology and treatment". The American Journal of Gastroenterology 83.5 (1988): 476-478.

8. Noriegga Maldonado. "Rapunzul syndrome. Gastro enterol". Hepatology 29.4 (2005): 259-261.

9. J Ahazam., et al. "Le trichobezoard gastrique: a propos d'un cas". Medecine du Maghreb (2008): 40-42.

10. Parekh VB Khaja M et al. Case Report: Trichobezoar in a 25 year old Autistic Female. New York Medical Journal.

11. Blam ME and Lichtenstein GR. "A new endoscopic technique for the removal of gastric phytobezoars". Gastrointestinal Endoscopy 52.3 (2000): 404-408.

12. Qureshi NH., et al. "Trichobezoar A condition to think of in case of mobile abdominal mass". Irish Medical Journal 85.2 (1992): 74 .

13. Deslypere JP., et al. "An unusual case of trichobezoar: The Rapunzel Syndrome". The American Journal of Gastroenterology 77.7 (1982): 467-470.

14. Sinzig M., et al. "Gastric trichobezoar with gastric ulcer: MR findings”. Pediatric Radiology 28.5 (1998): 296.

15. Mehta M and Patel RV. "Intussusception and intestinal perforations caused by multiple trichobezoars". Journal of Pediatric Surgery 27.9 (1992): 1234-1235.

16. Shawis RN and Doig CM. "Gastric trichobezoar associated with transient pancreatitis". Archives of Disease in Childhood 59.10 (1984): 994-995.

17. Katapadi M., et al. "Can a bezoar cause acute pancreatitis?". Journal of Clinical Gastroenterology 24.2 (1997): 120-121.

18. Hossenbocus A and Colin-Jones DG. "Trichobezoar, gastric polyposis, protein-losing gastroenteropathy and steatorrhoea". Gut 14.9 (1973): 730-732.
19. Chintapalli KN. "Gastric bezoar causing intramural pneumatosis". Journal of Clinical Gastroenterology 18.3 (1994): 264-265.

20. O'Soluvan MJ and Afrsci MB. "Trichobezoar". Journal of the Royal Society of Medicine 94 (2001): 68-70.

21. Rajaonarison P., et al. "Le trichobezoard, une entite clinique peu courante". Arch Inst Pasteur de Madagascar 67.2 (2001): 65-67.

22. Roche C., et al. "Trichobézoard: à propos de 5 observations". Arch Pediatr 12 (2005): 1608-1612.

23. Moujahid M., et al. "Un cas de trichobézoard gastrique". Pan African Medical Journal 9 (2011): 19.

24. Michaud L., et al. "Ingestion de corps étrangers chez l'enfant. Recommandations du Groupe francophone d'hépatologie, gastroentérologie et nutrition pédiatriques". Archives de Pédiatrie 16 (2009): 54-61.

25. Vaughan ED Jr., et al. "The Rapunzel syndrome. An unusual complication of intestinal bezoar". Surgery 63.3 (1968): 339343.

\section{Volume 2 Issue 7 September 2019}

(C) All rights are reserved by $\mathrm{R}$ Berraida., et $a l$. 